\title{
Filtering Characteristics of Hybrid Integrated Polymer and Compound Semiconductor Waveguides
}

\author{
Cem Ozturk, Andrew Huntington, Atilla Aydinli, Young Tae Byun, and Nadir Dagli, Member, IEEE
}

\begin{abstract}
This paper reports a study on a compact filter fabricated using hybrid integration of compound semiconductors and polymers. A GaAs epilayer is glued onto a polymer channel waveguide forming a highly asymmetrical directional coupler. This approach results in a narrow band filter due to very different dispersion characteristics of the compound semiconductor and the polymer materials. Furthermore, fiber coupling loss has been significantly reduced, since the input and output coupling is done through the polymer waveguide. Filtering characteristics can be engineered by changing the thickness and the length of the semiconductor epilayer. This can be done precisely using etch stop layers and noncritical lithography. The spectral response of such a filter can also be tuned electronically either using the electro-optic properties of the compound semiconductor or the thermo-optic properties of the polymer.
\end{abstract}

Index Terms-Bandstop filter, hybrid integration, tunable filter, wavelength division multiplexing (WDM).

\section{INTRODUCTION}

W IDESPREAD use of Internet and information technologies almost in all aspects of our daily lives resulted in an ever-increasing demand for bandwidth. Fiber optic communication networks have the potential to satisfy this demand. Wavelength division multiplexing (WDM) is a common way of increasing the capacity of fiber optic communication networks. Filtering is one of the most important elements of this technology. There is a long list of requirements for WDM filters, such as low insertion loss, low crosstalk, flat passband, wide tunability, fast tuning, and low cost. It is difficult to satisfy all these requirements using a single technology. Fiber Bragg gratings offer low insertion loss, good crosstalk [1]. But they are long due to low index contrast, environmentally sensitive, and hard to tune. They can be tuned mechanically or thermally, but this tuning is slow [2], [3]. Arrayed waveguide gratings combined with switches can offer fast tunable filtering with reasonable insertion loss, but they tend to be big [4]. Polymer gratings can be tuned slowly, but efficiently using thermal means [5].

Manuscript received September 26, 2001; revised May 8, 2002. This work was supported by the Defense Advanced Research Projects Agency (DARPA) through the Multidisciplinary Optical Switching Technology (MOST) Center, University of California at Santa Barbara.

C. Ozturk, A. Huntington, and N. Dagli are with the Electrical and Computer Engineering Department, University of California at Santa Barbara, Santa Barbara, CA 93106 USA (e-mail: dagli@ece.ucsb.edu).

A. Aydinli is with the Electrical and Computer Engineering Department, University of California at Santa Barbara, Santa Barbara, CA 93106 USA, and also with the Physics Department, Bilkent University, Ankara 06533, Turkey.

Y. T. Byun is with the Electrical and Computer Engineering Department, University of California at Santa Barbara, Santa Barbara, CA 93106 USA, and also with the Korean Institute of Science and Technology, Seoul 100-744, Korea.

Digital Object Identifier 10.1109/JLT.2002.800783
But they also tend to be long due to small index contrast [6]. Compound semiconductor gratings offer a large index contrast, hence, a compact device. Furthermore, electro-optic properties of compound semiconductors enable rapid tuning at least at the order of nanoseconds. However, coupling in an out of compound semiconductor waveguides using a cleaved optical fiber is lossy. One can deal with this coupling loss using mode transformers, but this requires a complex and expensive technology [7]. Another approach is to use mismatched directional couplers [8] and Mach Zehnder chains [9]. These devices are hard to tune in glass and polymer technology and suffer from the large coupling loss in compound semiconductor technology.

A possible solution to address the difficulties encountered in these technologies is a hybrid technology, which can be used to combine the best of different technologies. In this paper we present a study on the hybrid integration of polymers and compound semiconductors for WDM filtering applications. Utilization of commercially available polymers for the fabrication of fiber mode matched waveguides that serve as the input-output ports as well as the main waveguide for the device ensures low cost and overall low insertion loss. At the same time, utilization of compound semiconductors integrated with these waveguides as the filtering element ensures fast and wide tunability in a compact device. Moreover, novel processing technology needed to realize such a device can further be utilized for on chip integration of different WDM components.

In this paper, the principle of operation of the filter is described first. Then fabrication and the process details for the polymer waveguides and hybrid integration are given. This is followed by experimental results on the filter and its electronic tuning. Finally, conclusions are presented.

\section{DEVICE DESCRIPTION}

The generic schematic of the studied hybrid structure is shown in Fig. 1. The polymer waveguide is an inverted rib structure, while the GaAs slab waveguide is an epilayer removed from its growth substrate. Together they act as a highly asymmetric directional coupler. GaAs and polymer materials have very different indices of refraction $n_{\mathrm{GaAs}}$ and $n_{\text {Polymer }}$, respectively. Furthermore, their index dispersion is also very different. It is well-known that such asymmetric couplers act as narrow band optical filters [10]. The effective index of the polymer waveguide is around 1.5 while the index of GaAs is about 3.4. Since the GaAs slab waveguide have air and polymer as claddings, it is usually multimoded. The highest-order mode has an effective index slightly higher than the index of polymer cladding. Therefore, it can match the index of the polymer 


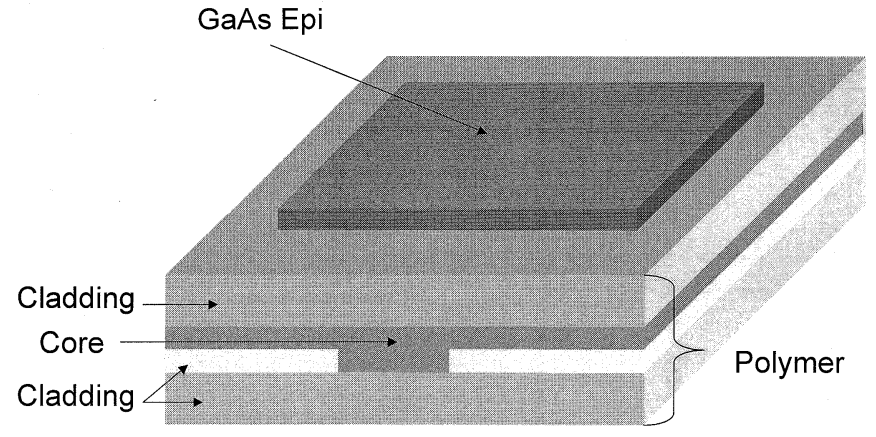

Fig. 1. Three-dimensional schematic of the hybrid filter.

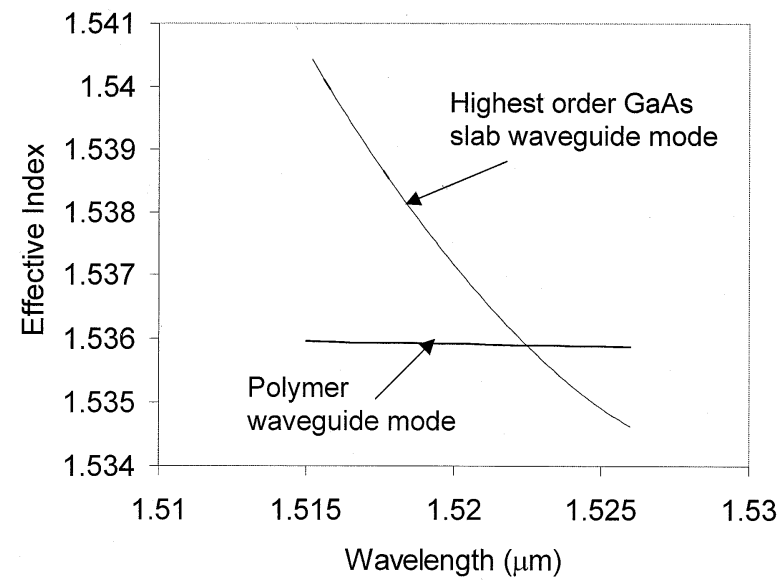

Fig. 2. Calculated effective index dispersion for the polymer mode, and the highest-order mode of the GaAs slab waveguide for TE polarization.

waveguide, resulting in strong coupling between the only mode of the single mode polymer waveguide and the highest-order mode of the GaAs waveguide. The effective index dispersion of the highest-order GaAs mode and the polymer waveguide mode are shown in Fig. 2 for TE polarization. Strong coupling occurs at the wavelength where the two dispersion curves intersect. Coupling to the other modes of the GaAs slab guide is negligible due to very large phase velocity mismatch. Depending on the coupling coefficient $\kappa$ and length $L$, different degrees of power transfer to the GaAs guide is possible. Since the dispersion characteristics of the two modes are very different, away from the mode matching condition, phase mismatch rapidly increases forcing the coupling to rapidly diminish with changing wavelength. This results in narrow filtering characteristics.

We can approximate the mode-matching wavelength, $\lambda$, simply by matching the individual effective refractive index, $n_{\text {eff }}^{\text {Poly }}$, of the polymer waveguide mode with the effective refractive index of the highest-order mode of the GaAs slab of thickness $t$. Then using well-known slab waveguide equations coupling occurs when

$$
\begin{array}{r}
T \sqrt{1-b}-\tan ^{-1} \sqrt{\frac{b}{1-b}}-\tan ^{-1} \sqrt{\frac{a+b}{1-b}}=m \pi \\
\text { for TE polarization }
\end{array}
$$

and

$$
\begin{aligned}
& T \sqrt{1-b}-\tan ^{-1}\left(\left(\frac{c+1}{c}\right)\left(\sqrt{\frac{b}{1-b}}\right)\right) \\
& -\tan ^{-1}\left(\left(\frac{c+1}{c}\right)\left(\sqrt{\frac{a+b}{1-b}}\right)\right)=m \pi \quad \text { for TM }
\end{aligned}
$$

polarization, where $T=(2 \pi t) /(\lambda) \sqrt{n_{\text {GaAs }}^{2}-n_{\text {Polymer }}^{2}}, a=$ $\left(n_{\text {Polymer }}^{2}-1\right) /\left(n_{\text {GaAs }}^{2}-n_{\text {Polymer }}^{2}\right), c=\left(n_{\text {Polymer }}^{2}\right) /\left(n_{\text {GaAs }}^{2}-\right.$ $\left.n_{\text {Polymer }}^{2}\right), b=\left(\left(n_{\text {eff }}^{\text {Poly }}\right)^{2}-n_{\text {Polymer }}^{2}\right) /\left(n_{\text {GaAs }}^{2}-n_{\text {Polymer }}^{2}\right)$, and $m$ is the index of the highest-order GaAs slab mode.

From (1) and (2), we can see that the tuning of the filtering characteristics is possible by tuning the electrical thickness of one of the waveguides. This requires changing either the thickness or index of refraction of one of the waveguides. The refractive index of the GaAs waveguide can be changed utilizing the well-known electro-optic properties of III-V compound semiconductors. The effective index of the polymer waveguide can also be changed utilizing thermo-optic effects. Therefore, the wavelength at which filtering occurs can be changed electronically.

The bandwidth of the filter depends on the $\kappa L$ product and the dispersion characteristics of the semiconductor and polymer waveguides. When $\kappa L=\pi / 2$ the filter bandwidth $\Delta \lambda$ can be expressed as [11], [12]

$$
\left.\Delta \lambda=0.8 \frac{\kappa \lambda_{0}^{2}}{\pi\left[\lambda \frac{\partial n_{\text {eff }}^{\text {Poly }}}{\partial \lambda}-\lambda \frac{\partial n_{\text {eff }}^{\mathrm{GaAs}}}{\partial \lambda}\right]}\right]_{\lambda=\lambda_{0}}
$$

where $\lambda_{0}$ is the center wavelength defined by (1), $n_{\text {eff }}^{\mathrm{GaAs}}$ and $n_{\text {eff }}^{\text {Poly }}$ are the effective indices of the highest-order GaAs and polymer waveguide modes, respectively. The denominator of this equation is the difference of the dispersion characteristics shown in Fig. 2. The very different dispersion characteristics of the polymer and semiconductor waveguides allow very narrow bandwidths.

Center wavelength of this filtering can also be tuned by changing the refractive indices of polymer or GaAs. In this case, the shift in the center wavelength is given as [11], [12]

or

$$
\frac{\Delta \lambda_{0}}{\lambda_{0}}=\frac{\Delta n_{\text {eff }}^{\mathrm{GaAs}}}{\left[\lambda \frac{\partial n_{\text {eff }}^{\mathrm{Poly}}}{\partial \lambda}-\lambda \frac{\partial n_{\text {eff }}^{\mathrm{GaAs}}}{\partial \lambda}\right]_{\lambda=\lambda_{0}}}
$$

$$
\frac{\Delta \lambda_{0}}{\lambda_{0}}=\frac{\Delta n_{\text {eff }}^{\text {Poly }}}{\left[\lambda \frac{\partial n_{\text {eff }}^{\text {Poly }}}{\partial \lambda}-\lambda \frac{\partial n_{\text {eff }}^{\mathrm{GaAs}}}{\partial \lambda}\right]_{\lambda=\lambda_{0}}}
$$

depending on if the GaAs or the polymer index change is the dominant index change. The same denominator as in (3) appears in this equation, showing that very narrow bandwidth filters fabricated this way are difficult to tune over a wide range, unless large index changes can be created.

It should be emphasized that the novelty of this paper is not the well-known asymmetric directional coupler idea, but the particular way it is implemented. Using a hybrid integration of polymers and semiconductors addresses the difficulties that 


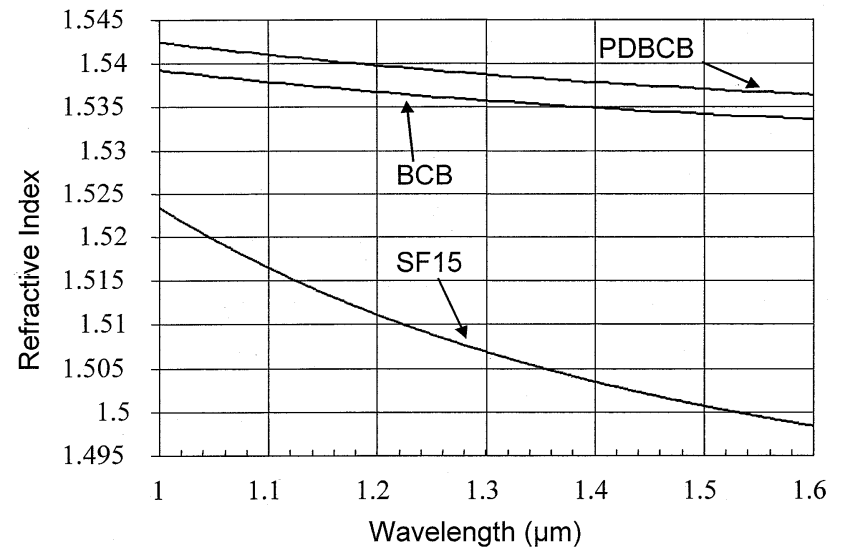

Fig. 3. Measured refractive index dispersion of the polymers used in fabrication.

plagued other technologies. This enables an electronically tunable narrow band filter. Furthermore, the fiber-to-fiber insertion loss is expected to be very low since the polymer waveguide mode shape and the index of refraction closely match that of the fiber.

\section{FABRICATION PROCESS}

\section{A. Polymer Waveguide Fabrication}

The polymers used in the fabrication of the polymer waveguides are benzocyclobutene (BCB) [13], photo-definable benzocyclobutene (PDBCB) [13], and polymethylglutarimide (PMGI), known as SF 15 [14]. These polymers are commercially available and are used in various aspects of microfabrication. Prior to fabrication, the refractive index dispersions for these polymers in the spectrum of interest were measured using the spectral ellipsometry technique. Results of this experiment are shown in Fig. 3. Of these polymers, PDBCB has the highest index and acts as the core material for the polymer waveguides. $\mathrm{BCB}$ not only acts as the lower and upper cladding, but also is the glue for further integration of these polymer waveguides with semiconductor waveguides. SF 15 is a deep-UV positive photoresist used to pattern the waveguide structure, and an essential part of the waveguide as the lateral cladding. The refractive indices of all the polymers used are very close to that of the fiber, minimizing facet reflections. Moreover the very small index step between BCB and PDBCB $(\Delta n \sim 0.003)$ helps to tailor the waveguide mode shape to match the mode of the single-mode fiber. This reduces the coupling losses significantly.

The process flow of the typical polymer waveguide fabrication and cross sectional profile of a partially finished polymer waveguide are shown in Fig. 4. The substrate is not an essential part of the waveguide, and any material with an optical quality surface can be used. In our experiments we use a semi-insulating (SI)-GaAs substrate, since optical quality facets can be formed very easily by cleaving. In the fabrication process first a thick layer of $\mathrm{BCB}$ is spun on the substrate and cured to form the lower cladding. It has also been found that a partial cure of the underlying polymer layers is essential, to enhance the sticking of the proceeding layers. A closed vacuum canister on a hot plate

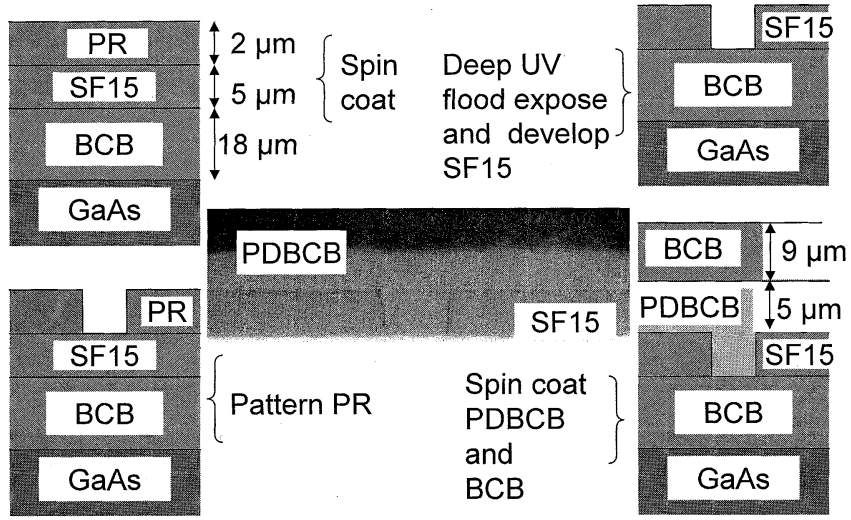

Fig. 4. Process flow of the polymer waveguide fabrication and the cross-sectional profile of a partially finished polymer waveguide.

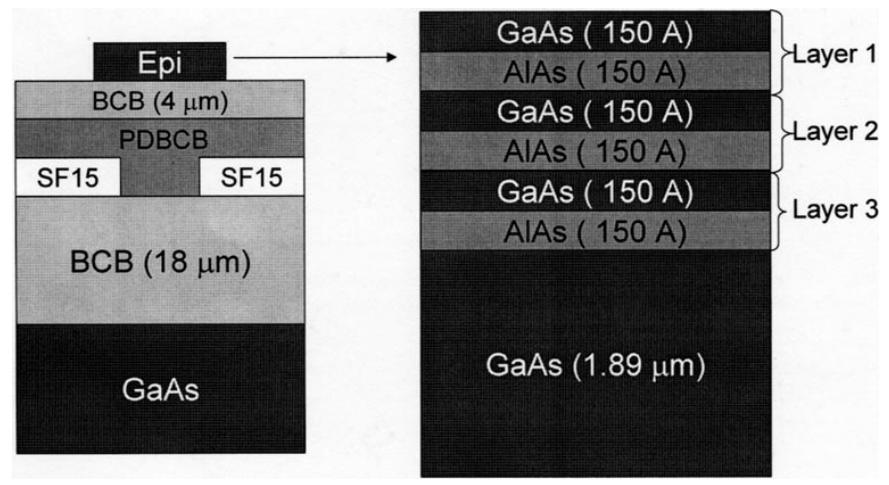

Fig. 5. Cross-sectional schematic of the hybrid structure, and the epilayer used in the fabrication.

is used for the partial curing of BCB. The temperature of the sample is monitored in-situ using a thermocouple. The curing scheme used involves a $10{ }^{\circ} \mathrm{C} / \mathrm{min}$ ramp to $210^{\circ} \mathrm{C}$, a $5 \mathrm{~min}$ hold, and a natural cool down yielding a curing rate of less than $80 \%$.

The patterning of the ridges is done using SF 15. A layer of SF 15 is spun on BCB and cured at $200^{\circ} \mathrm{C}$ for 2 min on a hot plate. The pattern is defined on top of SF 15 using AZ 4210 positive photoresist and conventional contact lithography. The photoresist is then used as the mask for the deep-UV flood exposure, and the ridge openings are then developed using SF 15 developer. After the removal of the photoresist mask, a layer of PDBCB is spun and partially cured to fill the ridge openings. PDBCB as well as BCB has very good gap filling properties after curing. Fig. 4 also shows the SEM picture of a partially completed polymer waveguide, which is taken after the curing of the PDBCB layer, illustrating the uniformity of the core layer.

The last step of the fabrication is the spinning of the upper cladding BCB layer, and the full cure of the whole polymer stack. A N-purged closed-box oven is used for the full-cure. The full-cure scheme used involves a $10{ }^{\circ} \mathrm{C} / \mathrm{min}$ ramp to $250{ }^{\circ} \mathrm{C}$, a $60 \mathrm{~min}$ hold, and natural cool down. The finished devices are then cleaved and mounted to form optical facets.

\section{B. Hybrid Integration}

The GaAs-AlGaAs epilayer structure used in the hybrid integration is shown in Fig. 5. It is grown on a SI-GaAs substrate 


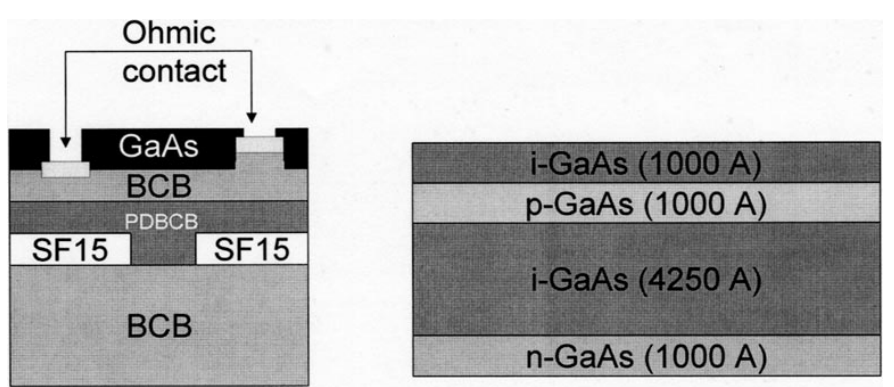

Fig. 6. Cross-sectional schematic of the electronically tunable filter, and the epilayer used in the fabrication.

using molecular beam epitaxy (MBE). This epilayer structure consists of an undoped 1.89- $\mu \mathrm{m}$ thick GaAs layer and three alternating $150-\AA$ thick AlAs-GaAs layers, which we will refer to as the tuning layers. There is a $0.2-\mu \mathrm{m}$ thick AlAs layer between the epilayer and the substrate, which is used as an etch-stop layer for the substrate removal etch. The integration of this structure with the polymer waveguides is done using the upper BCB cladding layer as the glue. The epilayer structure is brought into contact with the polymer waveguides episide down at $165^{\circ} \mathrm{C}$. It has been found that $\mathrm{BCB}$ loses its viscosity significantly between $160-170^{\circ} \mathrm{C}$, enabling the sample to float freely on its surface [15]. This is a key element for a void-free, uniform integration. It also enables us to align the edges of the transfer substrate and epigrown substrate, since the crystallographic alignment of the two samples is crucial to obtain optical quality facets by cleaving. The integration process is then finished by a full cure of the whole structure in $\mathrm{N}$-purged closed box oven. After the cure the growth substrate is etched using a 30:1 $\mathrm{H}_{2} \mathrm{O}_{2}: \mathrm{NH}_{4} \mathrm{OH}$ solution. This etch stops on the AlAs layer after the whole growth substrate is removed. The etch stop layer is then removed using 10:1 $\mathrm{H}_{2} \mathrm{O}: \mathrm{HF}$, leaving the thin epilayer bonded to the polymer waveguides. The device is then cleaved and mounted.

\section{Electronically Tunable Filter}

In addition to engineering a specific filter function, the ability to tune the filter response by electronic means is also very important. This can be done using the electro-optic properties of the semiconductor waveguide or thermo-optic properties of the polymer waveguide. For this purpose a different epilayer structure shown in Fig. 6 is designed and grown on a SI GaAs substrate. The GaAs slab waveguide in the finished device is a $p-i-n$ diode; hence, it can be either reverse or forward biased. Under reverse bias there is a vertical electric field between the $p$ and $n$ layers, which changes the refractive index of the $i$ region through the electro-optic effect. Under forward bias current flows between the doped layers through the $i$ region, and there is a carrier induced refractive index change in the $i$ region. Both effects can be utilized to investigate the feasibility and characteristics of electrical tuning.

In this case, the alignment of the polymer waveguides and the electrodes is needed. This is achieved by fabricating the device, including the polymer waveguides, on the epigrown substrate. In the fabrication first ohmic contacts to the $p$ and $n$ layers are formed using e-beam evaporation of $\mathrm{Ti}-\mathrm{Au}$ and $\mathrm{AuGe}-\mathrm{Ni}-\mathrm{Au}$

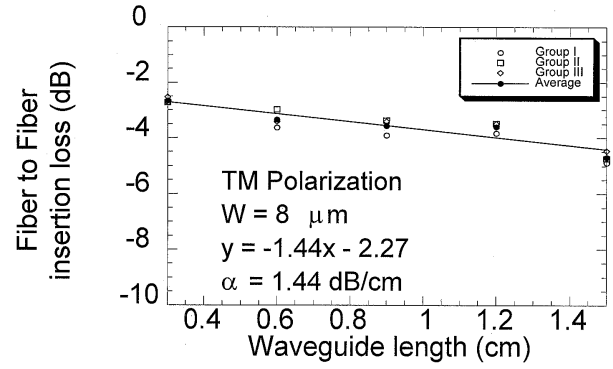

(a)

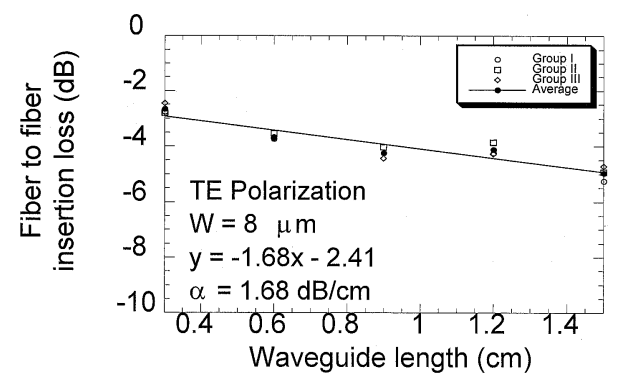

(b)

Fig. 7. Cutback loss measurement results for: (a) TE and (b) TM polarization of the fabricated polymer waveguides.

to the respective layers and annealing. The epilayer is then etched away leaving a $30-\mu \mathrm{m}$ wide GaAs slab waveguide. This step helps to minimize the device area, and to reduce the lateral radiation losses. The polymer waveguides are then fabricated on top of the GaAs slab waveguide as described earlier. Then the whole structure is transferred to a GaAs transfer substrate again using the $\mathrm{BCB}$ as the glue. The epigrown substrate is then etched away using the spray etching technique. The device is then cleaved and mounted.

\section{EXPERIMENTAL RESULTS}

\section{A. Polymer Waveguides}

Fig. 7 shows the fiber-to-fiber insertion loss of $8-\mu \mathrm{m}$ wide polymer waveguides as a function of the waveguide length for both TE and TM polarization at $1550 \mathrm{~nm}$. The measurements show that the polymer waveguides exhibit an average propagation loss of $1.6 \mathrm{~dB} / \mathrm{cm}$ and an average coupling loss of $1.1 \mathrm{~dB} /$ facet at $1550 \mathrm{~nm}$. The loss birefringence is very small. The low coupling loss is indicative of the good mode shape match of the polymer waveguide and the cleaved optical fiber. The results obtained are consistent with the earlier reported results on similar waveguides [16], [17].

\section{B. Hybrid Filter}

The spectral measurement of the hybrid filter shown in Fig. 5 is conducted using a tunable laser with a spectral range of $1500-1580 \mathrm{~nm}$. The fiber-to-fiber insertion loss of the hybrid filter for TM polarization is shown in Fig. 8(b). The length of the GaAs epilayer was $7.8 \mathrm{~mm}$, which satisfies $\kappa L=7(\pi / 2)$ to a good approximation. During the measurements thickness of the GaAs epilayer was changed by sequentially removing each tuning layer shown in Fig. 5 using selective etching. A 4:1 Citric Acid: $\mathrm{H}_{2} \mathrm{O}_{2}$ solution is used for the removal of the 


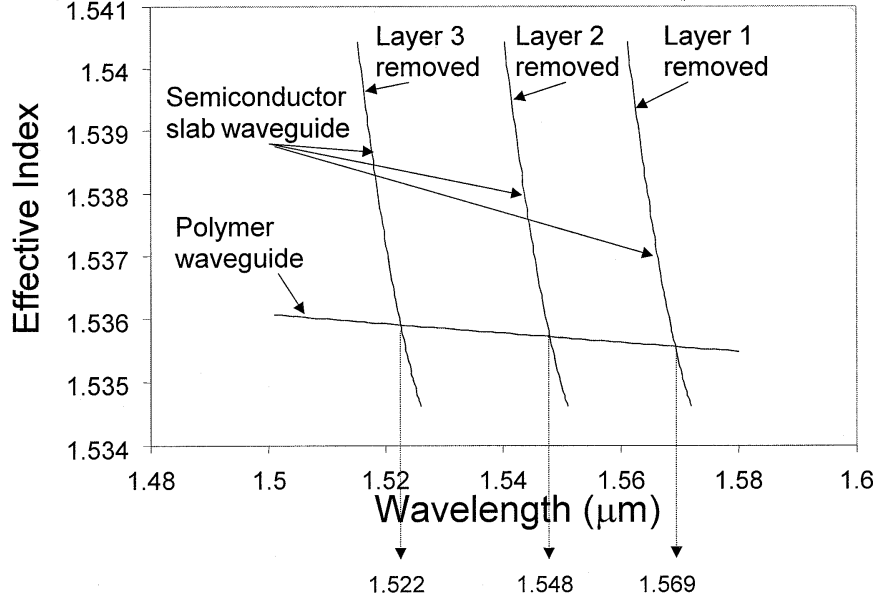

(a)

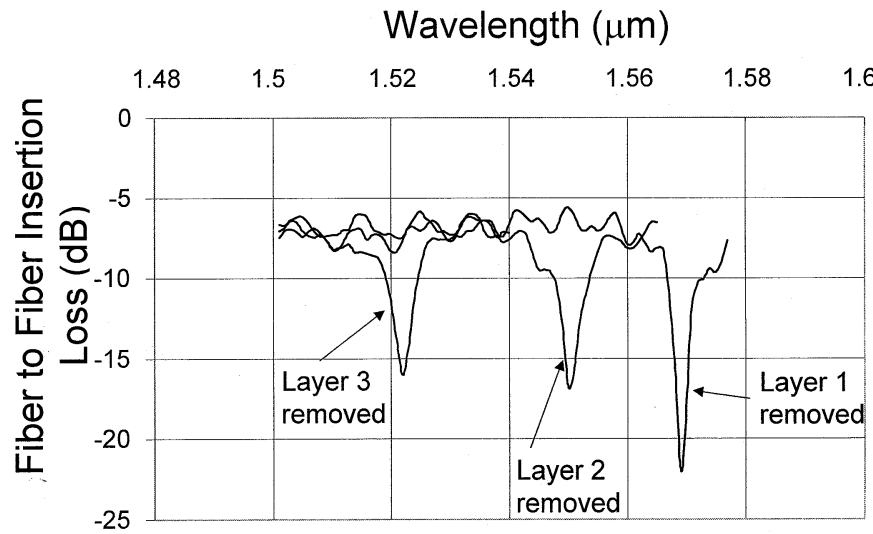

(b)

Fig. 8. (a) Calculated effective index of the polymer and semiconductor modes used to calculate the center wavelength for strong coupling. (b) Fiber-to-fiber insertion loss of the hybrid filter for different epilayer thickness for TM polarization.

GaAs, and a 10:1 $\mathrm{H}_{2} \mathrm{O}: \mathrm{HF}$ solution is used for AlAs. For each measurement a dip in the transmission through the polymer waveguide is observed. Hence, the device works as a band-stop filter similar to a long period fiber grating filter [18]. This dip is a result of the coupling between the polymer and semiconductor waveguides, which form a highly asymmetric directional coupler as described earlier. Since the effective index dispersion for the highest mode of the GaAs slab depends critically on the thickness, the spectral position of the transmission dip moves to shorter wavelengths with the removal of the tuning layers. As more tuning layers are removed the spectra also gets broader. The measurements show that the center wavelengths of filtering are 1569,1548 , and $1522 \mathrm{~nm}$ when tuning layers 1 , 2 , and 3 are removed sequentially. A comparison of this data, with the calculated results using (2) shows that they agree very well with the experimental results. Graphical representation of this solution is shown in Fig. 8(a). Any small deviation can be attributed to thickness nonuniformity that might have occurred during the very fast etching of the tuning layers. The broadening of the filter spectra can also be attributed to thickness nonuniformity along the length of the GaAs slab.

The fiber-to-fiber insertion loss is about $6.5 \mathrm{~dB}$. Although this loss is significantly lower than that of a semiconductor wave- guide filter, it is still higher than what is expected based on the polymer waveguide results given earlier. This is due to the extension of the GaAs epilayer all the way to the facets. Hence, coupling was not directly into the polymer waveguide. It was into the polymer waveguide capped with GaAs epilayer. This increases the coupling loss. This additional loss can be reduced significantly by tapering the GaAs epilayer using lithography.

Another observation is the polarization dependence of the filtering. For the experiment described in Fig. 8, TE spectra was flat. The position of the TE transmission dip calculated using (2) was outside the tuning range of the laser used in the experiments. Polarization dependence is a well-known feature for devices involving semiconductors. This issue can be mitigated either using birefringent epitaxial layers or polarization diversity schemes.

Since we can treat the coupled polymer waveguide GaAs slab system as a highly asymmetric directional coupler, we can use the coupled-mode theory to explain the characteristics of such a system. The degree of coupling depends on the $\kappa L$ product, where $\kappa$ is the coupling coefficient and $L$ is the length of the epilayer. We took advantage of this fact to experimentally determine the $\kappa$ value by curve fitting the experimental spectral response to well-known coupled mode equations for different epilayer lengths. For this purpose, devices are cleaved into different lengths after the removal of the third tuning layer and measured. The results of this approach showed that the $\kappa$ for the device is $\sim 14 \mathrm{~cm}^{-1}$. This experimental data agrees very well with the calculated data for shorter length devices. For longer length devices, the experimental data starts to deviate from the theory. This is mainly due to the lateral radiation into the GaAs slab, which is unaccounted for in the theoretical calculations.

These results show that such a hybrid device functions as a band-stop filter. One can also engineer the spectral shape of the filter by changing the thickness of the GaAs epilayer along the polymer waveguide. This can be done as described in this paper, namely using AlAs etch stop layers and GaAs layers of different thickness. Since the thicknesses of these layers are controlled during epitaxial growth, the control of the transmission dip wavelengths can be done very accurately. One can control the degree of the transmission by controlling the length of the epilayer sections having different epilayer thickness. The result could be a desired filtering shape. Such a device, for example, can be used as a compact gain-equalizing filter for erbium doped fiber amplifiers.

\section{Electrical Tuning}

The spectral measurement of the device under different bias conditions is again conducted using a tunable laser with a spectral range of 1500-1580 nm. Fig. 9 shows the fiber-to-fiber transmission through the polymer waveguide. In this case, fiber-to-fiber insertion loss is about $5 \mathrm{~dB}$. As expected a transmission dip is observed. Center wavelength of the dip shifts linearly to shorter wavelengths with applied reverse voltage. The average spectral shift is found to be $0.1 \mathrm{~nm} / \mathrm{V}$ for this particular design. In this case, the shift is due to the refractive index change through the linear electro-optic effect. 


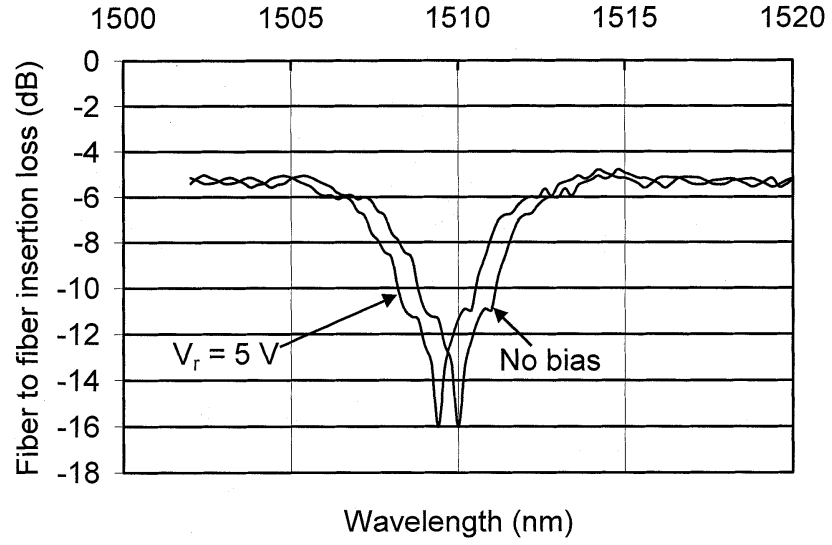

Fig. 9. Fiber-to-fiber insertion loss of the electronically tunable filter under reverse bias for TE polarization.

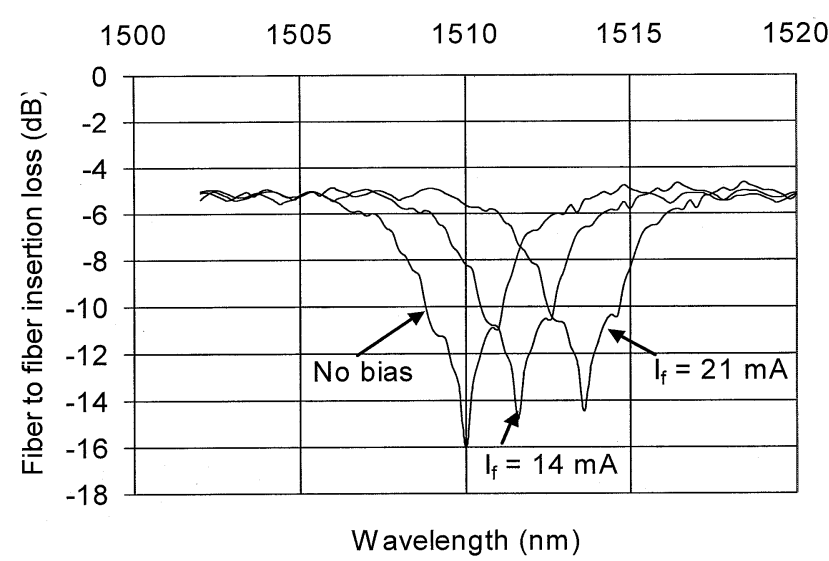

Fig. 10. Fiber-to-fiber insertion loss of the electronically tunable filter under forward bias for TE polarization.

The effective index change of the highest-order GaAs slab waveguide mode can be expressed as

$$
\Delta n_{\mathrm{eff}}^{\mathrm{GaAs}}=\frac{1}{2} n_{\mathrm{GaAs}}^{3} r_{41} \frac{V}{d_{i}} \Gamma
$$

where $n_{\mathrm{GaAs}}$ is the index of GaAs, $r_{41}$ is the bulk electro-optic coefficient of GaAs, $d_{i}$ is the thickness of the $i$ region, and $\Gamma$ is the overlap of the highest-order GaAs mode with the $i$ layer. In turn the shift in the transmission dip corresponding to $\Delta n_{\mathrm{eff}}^{\mathrm{GaAs}}$ can be calculated using (4). Using these equations, we calculate an expected spectral shift of $0.09 \mathrm{~nm} / \mathrm{V}$, which is in reasonable agreement with the experimental value. The reason for such a small tuning range is twofold. First, $r_{41} \approx 1.6 \times 10^{-12} \mathrm{~m} / \mathrm{V}$ for bulk GaAs is very small. Second, large index dispersion difference between the polymer and GaAs waveguides makes tuning difficult as seen in (4), although it makes the filter bandwidth narrow as indicated by (3). Larger tuning ranges can be achieved using different material designs, such as quantum wells, and different dimensions.

Fig. 10 shows the fiber-to-fiber transmission of the filter under forward bias. As bias current increases the transmission dip moves to longer wavelengths, while the spectral shape remains unchanged. Carrier injection reduces the refractive index in GaAs [19]. This would in turn reduce the effective index of the highest-order mode. An examination of Fig. 2 reveals that the dip position would move to shorter wavelengths. But, the observed shift of the filter spectra to the longer wavelengths is not consistent with the expected effect of carrier induced refractive index change of the GaAs slab. Furthermore, the applied current to the $p-i-n$ structure is far below the amount needed to change the refractive index of the semiconductor layer to yield the measured tuning range. The only other effect that would cause a shift to the longer wavelengths is a negative change in the effective refractive index of the polymer waveguide. The thermo-optic coefficients for all the polymers used in the fabrication of the device are negative, implying an increase in temperature would decrease their refractive indices. In our device the heat dissipated through the semiconductor layer via current injection is transferred to the polymer waveguide, decreasing the effective refractive index of the polymer mode. The measurements show that the shift of the filter spectra to longer wavelengths is linear with the power dissipated in the semiconductor. The shift of the transmission dip is $0.1 \mathrm{~nm} / \mathrm{mW}$. In this case, heating of the polymer waveguide is not very efficient. One can increase the efficiency of the tuning by fabricating built in heaters next to the polymer waveguide. This makes thermoelectric tuning very desirable for broad tuning applications.

\section{CONCLUSION}

In this paper, a novel technology to fabricate an asymmetric directional coupler filter was presented. We reported a compact filter fabricated using hybrid integration of compound semiconductors and polymers. A GaAs slab waveguide was integrated with a polymer channel waveguide by gluing the partially fabricated epilayer on a growth substrate onto the polymer waveguide. In this process, the upper cladding of the polymer waveguide was used as glue. Then the growth substrate was removed using a selective etch resulting in a highly asymmetrical directional coupler. This approach resulted in a narrow band filter due to very different dispersion characteristics of the compound semiconductor and the polymer materials. Furthermore, fiber coupling loss was significantly reduced, since the input and output coupling was done through the polymer waveguide. It was shown that the center filter wavelength of such a device could be tuned by changing the thickness of the GaAs epilayer slightly. Furthermore the filtering strength can be controlled by changing the length of the epilayer. As a result the filtering characteristics of such a filter can be engineered by changing the thickness and the length of the semiconductor epilayer. This can be done precisely using etch stop layers and noncritical lithography. The spectral response of such a filter was also tuned electronically both using the electro-optic properties of the compound semiconductor and the thermo-optic properties of the polymer.

\section{REFERENCES}

[1] K. O. Hill and G. Meltz, "Fiber Bragg grating technology fundamentals and overview," J. Lightwave Technol., vol. 15, pp. 1263-1276, Aug. 1997.

[2] T. Inui, T. Komukai, and M. Nakazawa, "Highly efficient tunable fiber Bragg grating filters using multilayer piezoelectric transducers," Opt. Commun., vol. 190, no. 1-6, pp. 1-4, Apr. 1, 2001. 
[3] A. A. Abramov, B. J. Eggleton, J. A. Rogers, R. P. Espindola, A. Hale, R. S. Windeler, and T. A. Strasser, "Electrically tunable efficient broad-band fiber filter," IEEE Photon. Technol. Lett., vol. 11, pp. 445-447, Apr. 1999.

[4] M. K. Smit and C. van Dam, "PHASAR-based WDM-devices: Principles, design and applications," IEEE J. Quantum Electron., vol. 2, pp. 236-250, Feb. 1996.

[5] H. Y. Liu, G. D. Peng, and P. L. Chu, "Thermal tuning of polymer optical fiber Bragg gratings,” IEEE Photon. Technol. Lett., vol. 13, pp. 824-826, Aug. 2001.

[6] Z. Xiong, G. D. Peng, B. Wu, and P. L. Chu, "Highly tunable Bragg gratings in single mode polymer optical fibers," IEEE Photon. Technol. Lett., vol. 11, pp. 352-354, Mar. 1999.

[7] N. Yoshimoto, K. Kawano, H. Takeuchi, S. Kondo, and Y. Noguchi, "Spot size convertors using InP/InAlAs multi quantum well waveguides for low loss single mode coupling," Electron. Lett., vol. 28, no. 17, pp. 1610-1611, Aug. 1992.

[8] R. Alferness, T. Koch, L. Buhl, F. Storz, F. Heismann, and M. Martyak, "Grating assisted InGaAsP/InP vertical codirectional coupler filter," Appl. Phys. Lett., vol. 55, pp. 2011-2013, Nov. 1989.

[9] M. Kuznetsov, "Cascaded coupler Mach-Zehnder channel dropping filters for wavelength-division-multiplexed optical systems," J. Lightwave Technol., vol. 12, pp. 226-230, Feb. 1994.

[10] R. C. Alferness and P. S. Cross, "Filter characteristics of codirectionally coupled waveguides with weighted coupling," IEEE J. Quantum Electron., vol. QE-14, pp. 843-847, 1978.

[11] R. C. Alferness, U. Koren, L. L. Buhl, B. I. Miller, M. G. Young, T. L. Koch, G. Raybon, and C. A. Burrus, "Broadly tunable InGaAsP/InP laser based on a vertical coupler filter with $57 \mathrm{~nm}$ tuning range," Appl. Phys. Lett., vol. 60, pp. 3209-3211, June 1992.

[12] Z. M. Chuang and L. A. Coldren, "Design of widely tunable semiconductor lasers using grating-assisted codirectional-coupler filters," IEEE J. Quantum Electron., vol. QE-29, pp. 1071-1080, Apr. 1993.

[13] "Dow Plastics," The Dow Chemical Company, Midland, MI, Form No.: 296-01 211-493NP\&M.

[14] "PMGI SF," Series Resists, MicroChem Corporation, Newton, MA.

[15] S. R. Sakamoto, A. Jackson, and N. Dagli, "Substrate removed GaAs/AlGaAs modulator," IEEE Photon. Technol. Lett., vol. 11, pp. 1244-1246, Oct. 1999.

[16] C. F. Kane and R. R. Krchnavek, "Benzocyclobutene optical waveguides," IEEE Photon. Technol. Lett., vol. 7, no. 5, pp. 535-537, May 1995.

[17] G. Fishbeck, R. Moosburger, C. Kostrzewa, A. Achen, and K. Petermann, "Single mode optical waveguides using a high temperature stable polymer with low losses in the $1.55 \mu \mathrm{m}$ range," Electron. Lett., vol. 33, no. 6, pp. 518-519, Mar. 13, 1997.

[18] A. M. Vengsarkar, P. J. Lemaire, J. B. Judkins, V. Bhatia, T. Erdogan, and J. E. Sipe, "Long-period fiber gratings as band-rejection filters," $J$. Lightwave Technol., vol. 14, pp. 58-65, 1996.

[19] B. Bennett, R. Soref, and J. Alamo, "Carrier-induced change in rrefractive index of InP, GaAs, and InGaAsP," IEEE J. Quantum Electron., vol. QE-26, pp. 113-122, Jan. 1987.

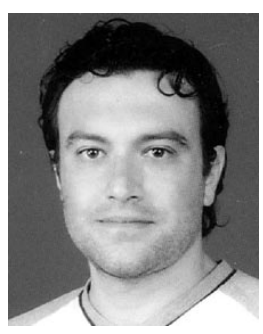

Cem Ozturk was born on November 27, 1972, in Izmir, Turkey. He received the B.S. degree in electrical and electronics engineering from Bogazici University, Istanbul, Turkey, in 1995 and the M.S. and $\mathrm{Ph} . \mathrm{D}$. degrees in electrical and computer engineering from the University of California, Santa Barbara, in 1997 and 2002, respectively. His graduate studies focused on the development of tunable hybrid polymercompound semiconductor devices for WDM applications.

He is currently employed as an Assistant Research Engineer at the Electrical and Computer Engineering Department, University of California, Santa Barbara. His current research interests include the development of tunable hybrid microdisc resonator devices for the realization of ultracompact optoelectronic components.
Andrew Huntington, photograph and biography not available at the time of publication.

Atilla Aydinli, photograph and biography not available at the time of publication.

Young Tae Byun, photograph and biography not available at the time of publication.

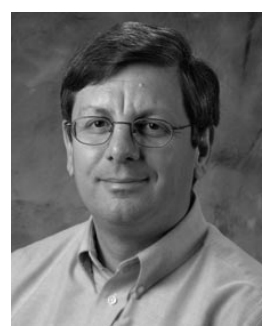

Nadir Dagli (S'77-M'87) received the B.S. and M.S. degrees from the Middle East Technical University, Ankara, Turkey, in 1976 and 1979, respectively, and the Ph.D. degree, from the Massachusetts Institute of Technology, Cambridge, in 1986, all in electrical engineering. During his Ph.D. research he worked on the design, fabrication, and modeling of guided wave integrated optical components in III-V compound semiconductors. He also worked on III-V materials preparation by LPE and the modeling and analysis of heterojunction bipolar transistor for microwave and millimeter-wave applications.

In 1986, he joined the Electrical and Computer Engineering Department, University of California, Santa Barbara, where he is currently a Professor. His current interests are design, fabrication, and modeling of guided-wave components for optical integrated circuits, ultrafast electrooptic modulators and WDM components, calculations on the optical properties of quantum wires, experimental study of novel quantum devices based on ballistic transport in quantum wires.

Dr. Dagli is the Editor-in-Chief of IEEE PHOTONICS TECHNOLOGY LETTERS. Currently, he is serving on the Microwave Photonics Committee of IEEE Lasers, Electro Optics Society Annual Meeting, International Topical Meeting on Microwave Photonics, Optoelectronic Device and Optical System Modeling Subcommittee of The Fifth Pacific Rim Conference on Lasers and Electro-Optics. 